\title{
Providing Context-Based Adaptation in Collaborative Virtual Environments and Video Games
}

\author{
Anastasiia Beznosyk ${ }^{1}$, Johanna Renny Octavia Hariandja ${ }^{1,2}$, Karin Coninxa ${ }^{1}$, Peter Quax ${ }^{1}$, \\ Wim Lamotte ${ }^{1}$ \\ ${ }^{1}$ Hasselt University - Expertise Centre for Digital Media - tUL - iMinds, \\ Wetenschapspark 2, 3590 Diepenbeek, Belgium \\ ${ }^{2}$ Parahyangan Catholic University \\ Ciumbuleuit 94, Bandung 40141, Indonesia
}

\begin{abstract}
This paper addresses our effort in investigating context-based adaptation as a prospective solution to enhance group interaction in collaborative virtual environments and video games. Through our work, we aim to provide adaptation of assigning users' roles based on the context by users when collaboratively interacting in a virtual world. We first describe a user experiment in a fully controlled environment to assess whether or not the adaptation results in improved group collaboration in virtual environments. Afterwards, we investigate to what extent the suggested approach for adaptation can be applied in a real-life scenario. In particular, we identify some of the challenges that arise when employing role-based adaptation in existing multiplayer video games. From the experiments, we learned that the context-based adaptation of assigning roles to users improves the group performance.
\end{abstract}

\section{Introduction}

Collaboration in virtual environments has become more popular during the last decades due to the rise of Massive Multiplayer Online Role-Playing Games (MMORPG) such as World of Warcraft ${ }^{1}$ and virtual communities such as Second Life ${ }^{2}$. Collaboratively interacting in virtual environments has its own challenges compared to other types of collaborative work. A collaborative virtual environment involves a group of users performing highly interactive tasks with the use of three-dimensional (3D) user interfaces, while trying to achieve effective and efficient collaboration. Another complexity of working in collaborative virtual environments is the variety of $3 \mathrm{D}$ interaction tasks and techniques. The performance and preferences of every single user may differ, which in the end may influence the group collaboration. Providing adaptation according to the context of users is considered beneficial to improve

\footnotetext{
${ }^{1} \mathrm{http}: / /$ www.worldofwarcraft.com

${ }^{2}$ http://www.secondllife.com
}

their interaction in virtual environments, not only individually but also as a group collaboratively. Adaptation may play an important role in elevating user performance in dynamically changing virtual worlds. Therefore, to enhance group interaction in collaborative virtual environments, we envisage that providing adaptive interaction, according to the individual context of users, would be a prospective solution.

Through our work, we would like to investigate if context-based adaptation leads to enhanced group interaction in collaborative virtual environments. In particular, we propose an approach of assigning a certain role and device to the users based on their performance. After analyzing this approach for a general task in a controlled virtual environment, we investigate its potential in a real-life scenario based on the example of an existing commercial video game. Here, we identify some of the challenges that arise when employing the suggested approach for adaptation in games. The results of our studies reveal the advantages of applying adaptation in collaborative virtual environments and video games.

\section{Background}

\subsection{Context-based Adaptation in Virtual Environments}

Interacting in virtual environments is mostly associated with complex interaction where users have to perform complicated tasks with highly interactive 3D user interfaces. Different user characteristics influence user interaction in virtual environments and lead to performance deviation among users. Adaptive user interfaces can be considered as one way to accommodate these individual differences and level up users' performance [1]. Integrating adaptation into virtual environments, by providing adaptive user interfaces according to users and other contexts, is considered as a key to comply with user's differences and enhance user interaction.

We acknowledge the importance of providing adaptation in virtual environments to enhance user 
interaction. The adaptation should take place according to the current context, for instance the environment conditions of the virtual world and the preferences and abilities of the users.

Over the past few years, a considerable amount of research has been done in context-aware computing. Dey [2] described the general definition of context, which is likely the most popularly used in every HCI field: "Context is any information that can be used to characterize the situation of an entity. An entity is a person, place, or object that is considered relevant to the interaction between the user and the application, including the user and the applications themselves."

The use of context for making decisions about adaptation for a variety of user interfaces has raised significant interest in the research community. For instance, many web applications utilize personalization and adaptation based on the user's history and preferences [3].

Not much research has been done on adaptation in virtual environments compared to WIMP applications. Nonetheless, few works have yielded adaptivity of interaction in virtual environments by means of learning user behavior and preferred method of interaction [4]. In [5], the authors have investigated an approach for detecting and switching context for contextual interaction in virtual environments, and integrating adaptation in virtual environments using a context-aware design process. In those works context was defined by the available input and output devices, and also external parameters such as the experience level of the user, whether or not there are collaborative partners in the environment or even the pose of the user (sitting or standing).

In [6], the author has experimented with providing adaptation such as switching between interaction techniques (based on personal preference) and adapting the interaction technique itself (based on motor ability). Adaptation of the user interfaces and interactions to the context of used devices, the user's needs and the environment has also been studied in [7].

\subsection{Adaptation in Video Games}

Video games are an increasingly popular kind of virtual environments. The amount of video games in the market constantly grows. So does the number of players, whose characteristics, needs and preferences are getting more and more diverse. Therefore, to ensure a longer time a game is being played, producers need to take into account the differences among potential players and create conditions that motivate the widest possible range of users to keep playing. This triggers a need for tailoring games to individual playing experiences, making them adaptive and personalized to the characteristics of each player. In particular, this can be achieved by modeling of player experience, an approach which has been identified as one of the key components to achieve a high level of player satisfaction [8]. The player model incorporates any relevant information, such as playing style and current emotional and cognitive state, which are used for steering the adaptive components of a game (e.g. adaptation of task, character, game mechanics, sound, difficulty, etc.).

Player modeling and adaptive technologies facilitate an improved player-centered game design by providing a more appropriate level of challenge, a smoother learning curve, and enhance the game-play experience for individual players. A substantial amount of research has successfully attempted to incorporate the adaptation in games such as the modification of difficulty levels [9], enemy's behavior [10], or graphic elements of the game environment itself [11]. While most of these research initiatives focus on the adaptation in single-user environments, our work investigates adaptation in a collaborative setting, discussed further in Section 3.

\subsection{Collaboration in Virtual Environments}

Collaboration in virtual environments has been studied quite extensively for the last few decades. Many collaborative virtual environments (CVEs) have come into existence. However, most of them have limited interaction possibilities and have not widely supported highly interactive activities yet. Nevertheless, there are few studies [12,13] which have investigated people collaborating to perform interactive tasks. For example, in [13] all participants had to complete different subtasks in order to achieve the task goal. The variation of performance based on the available output devices was investigated in this work. In our opinion it is interesting to see if the adaptation of the tasks based on the devices would increase the performance, as explored further in the article.

Another example of highly interactive collaboration can be found in [12] which investigated the collaboration of two participants on a Rubik's cube task. The authors compared performance between a real and an immersed setup, which showed to be almost the same. Their analysis revealed higher rates of these two setups comparing to the desktop one. It also showed that the participant using the desktop contributed less to the task and did not enjoy the collaboration. Based on this, it may be interesting to improve collaboration by adjusting the desktop user performance by assigning him certain activities that can be done easily by him. This can be seen as one example where adaptation based on the devices and performance can enhance the collaboration of several people.

The research presented in [14] investigated different factors that influence collaboration in a 
virtual environment, namely communication and variety of input devices. The experiments showed that there is a correlation between the devices used with the roles taken. It was natural that participants divided their roles based on their convenience with the device. But this division was done explicitly only when communication was allowed. For no communication cases, it was not possible to divide the roles explicitly. We believe that this issue can be solved by automatic adaptation of users' roles in collaborative virtual environments.

\section{Context-Adaptive Collaboration in Virtual Environments and Multiplayer Games}

Numerous works in the field of CSCW and mobile applications have tried to provide adaptation based on context to improve group collaboration [15]. However, context-adaptive interaction has received less attention in multi-user virtual environments.

One phenomenon of recent CVEs is their rapidly increased popularity and ability to unite thousands of people all over the world. The main aim for developers now is to support interaction between these users, which can be achieved for example by providing different services based on their context [16]. Another approach to provide enhanced collaboration is context-aware communication support for remote gamers [17], which can improve a gaming experience by integrating voice interaction between users based on the game context.

To our knowledge, there are only very few works which describe how collaborative virtual environments or multiplayer games can benefit from adaptation based on the users' context. In [18] a collaborative virtual environment for training is presented, where different elements (virtual world, virtual humans' behavior, scenario, etc.) can be adapted to a specific training situation. In [19], the authors suggest the use of adaptation in two-player competitive games such as tennis and air hockey, which allows both players enjoying the game in spite of their different levels. More specifically, if a player is playing too badly (the game is too difficult for him), the game gets easier for him so he does not get frustrated. If a player is playing too well (the game is too easy for him), the game gets more difficult for him so he does not get bored. Adaptation happens according to the context of user performance (i.c. amount of hits done by each player).

In [20], an attempt to integrate adaptation based on a user context has been made in the domain of multiplayer video games. Here, the adaptation was provided based on an individual player's performance and preferences, while interacting in a 3D game. The authors have shown that the player experience in collaborative games can be significantly improved by adapting the means of interaction based on the players' model. Four different approaches to build a player model were suggested based on the game goal.

The aim of collaborative virtual environments should be to achieve group goals, not individual goals of each user involved in the collaboration. Group interaction and performance may be enhanced by integrating adaptation into collaborative virtual environments. To provide adaptation in a collaborative setting, we consider an approach of employing a group context which is based on the individual context of users involved in the group collaboration. Therefore, we would like to investigate the possibility of combining individual contexts into a group context. We believe that singleuser based adaptation for a collaborative environment, where adaptation is performed separately for each user involved in the collaboration, does not lead to an adaptive collaborative environment. Additionally, it is essential to examine the integration of exogenous context factors (e.g. location, device used) with endogenous ones (e.g. users' roles, experiences) to provide better and more adequate adaptation in collaborative environments.

The goal of the work presented in this article is twofold. First of all, we aim to investigate how the adaptation can be applied in collaborative virtual environments in general. We investigate a combination of endogenous and exogenous factors to assess if context-based adaptation leads to enhanced group interaction (i.e. higher productivity) in collaborative virtual environments. For this purpose, we carry out an experiment to investigate how adaptation based on the context of the users' roles and devices used can benefit collaboration within a virtual world. While performing a collaborative $3 \mathrm{D}$ puzzle solving task, users' roles are divided based on their individual performance in executing a 3D object manipulation task.

Secondly, we investigate the applicability of the same approach in a specific application domain, namely video games. Based on the example of the existing commercial game Trine ${ }^{3}$, we identify what challenges occur when integrating the role-based adaptation in a real-life scenario.

\section{Proposed User Modeling Approach}

In our work, we propose adaptation of users' roles such that it will result in a better group interaction. To determine the adaptation, we propose an approach to construct a collaborative user model based on users' individual performances. The purpose of this user modeling approach is to come up

\footnotetext{
${ }^{3}$ http://trine-thegame.com
} 
with the best prediction of role combination for providing adaptation by assigning users' roles (i.e. deciding which roles are appropriate for the users) to enhance the group interaction. In this work, we suggest a 'minimum time' approach as a way to build the collaborative user model. In this approach, we estimate the task completion time for every possible role combination and then select the combination which gives the minimum estimated total time as the best role combination. To estimate the task completion time, firstly, we calculate for every user the time to perform each role separately. Then for all combinations of roles, we calculate the total time to perform both roles collaboratively. To validate this approach, we compare the findings based on our model with the actual role-based performance. Besides validation of our approach, we also study whether or not role-based collaboration enhances the group performance.

The presented approach has been applied in two experiments: for a general collaborative virtual environment and in a multiplayer video game. The differences that have occurred due to the different task nature during the user performance modeling are discussed in the following sections.

\section{Experiment I: Adaptation in a Collaborative Virtual Environment}

As a first step we want to investigate how to enhance group interaction by providing contextbased adaptation in a general collaborative virtual environment, not a specific application like a commercial game investigated later in Section 6. The adaptation is determined based on the context of users' role and device used when collaboratively interacting in a virtual world. To achieve this, we conduct an experiment to investigate the performance of users in pairs while executing an interactive 3D puzzle solving task, which is considered to be highly collaborative. The experiment is designed based on [14] which showed that users explicitly divided their roles during collaboration based on their convenience with the device being used. With our experiment, we aim an improved group interaction by constructing a collaborative user model that suggests the adaptation of assigning users' roles based on their individual performance when executing a certain task (e.g. rotation, translation) using a certain device (e.g. SpaceMouse, Phantom). Through this experiment, we would also like to validate our proposed approach in constructing the collaborative user model described in Section 4.

\subsection{Hypotheses}

For the best combinations predicted by the collaborative user model, three hypotheses were formulated in this experiment: (1) The estimated task completion time based on the model will not differ from the actual task completion time, (2) The estimated task completion time based on the model will be lower than the free-collaboration task completion time, and (3) The actual task completion time will be lower than the free-collaboration task completion time.

\subsection{Participants}

Ten randomly coupled pairs of unpaid volunteers (four females and sixteen males, 23 to 34 years old) participated in the experiment. Most of the participants had little experience with a virtual environment and also with the devices involved. All participants were right-handed and used their dominant hand to operate the device during the experiment.

\subsection{Apparatus}

For the experiment, two input devices, a SpaceMouse and a Phantom, were available for each user. Two desktop computers with 19 inches displays were used in the experiment. During the experiment, participants were located in the same room and close to each other, however, they were seated such that it was impossible to see each other's screen. Figure 1 illustrates the experiment apparatus and settings.

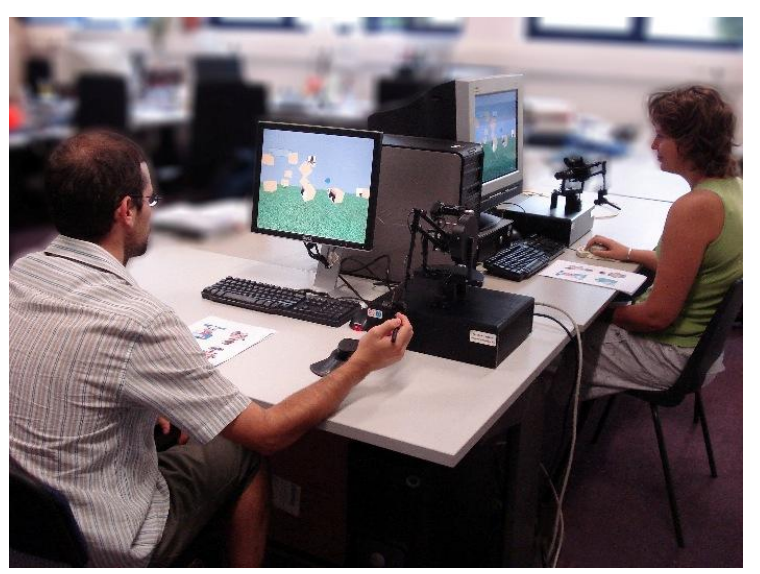

Figure 1. The setup in the first experiment.

\subsection{Design and procedure}

The experiment consisted of three parts: an individual session, a free collaborative session, and a role-dependent collaborative session. The first session aimed to gather the data of each user's performance individually, with regards to the combination of roles and devices. The second and third sessions aimed to gather the data of the users' 
performance in pairs; in the second session there was no division of roles while in the third session the division of roles was specifically defined. Two roles were defined in the experiment: rotation and translation, which were performed using either SpaceMouse or Phantom. Table 1 presents the complete overview of the experiment sessions.

A within-subject ${ }^{4}$ design was used for the collaborative sessions of the experiment. For the free collaborative session, the independent variable was the device used. For the role-dependent collaborative session, the independent variable was the combination of role and device, which order was counterbalanced. Task completion time was measured as the dependent variable.

Table 1. Sessions in the first experiment (puzzle solving task).

\begin{tabular}{|c|c|c|}
\hline & Participant 1 & Participant 2 \\
\hline \multirow{4}{*}{ 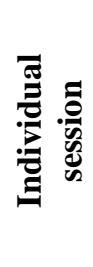 } & Rotation SpaceMouse & Rotation SpaceMouse \\
\hline & $\begin{array}{l}\text { Translation } \\
\text { SpaceMouse }\end{array}$ & $\begin{array}{l}\text { Translation } \\
\text { SpaceMouse }\end{array}$ \\
\hline & Rotation Phantom & Rotation Phantom \\
\hline & Translation Phantom & Translation Phantom \\
\hline \multirow{2}{*}{ 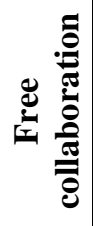 } & Free SpaceMouse & Free Phantom \\
\hline & Free Phantom & Free SpaceMouse \\
\hline \multirow{4}{*}{ 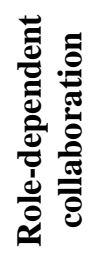 } & Rotation SpaceMouse & Translation Phantom \\
\hline & $\begin{array}{l}\text { Translation } \\
\text { SpaceMouse }\end{array}$ & Rotation Phantom \\
\hline & Rotation Phantom & $\begin{array}{l}\text { Translation } \\
\text { SpaceMouse } \\
\end{array}$ \\
\hline & Translation Phantom & Rotation SpaceMouse \\
\hline
\end{tabular}

We asked every pair to complete ten puzzle solving tasks. Each puzzle consists of 12 cubes representing a picture, where one cube was already placed to serve as a visual guide. The cubes, with a part of the picture on one side, were dispersed in the virtual environment (see Figure 2).

During the experiment, participants needed to indicate the starting and ending time of the session by selecting the menu items ("Rotation/Translation" to start the first and third session, "Start" to begin the second session, and "Finish" to end all sessions as soon as the task was completed). Participants were allowed to talk during the experiment. At the end of each session, we asked the participants to fill out a questionnaire about their experience and preference.

\footnotetext{
${ }^{4}$ Subjects are defined as pairs
}

Each pair completed the experiment in 60 minutes on average.

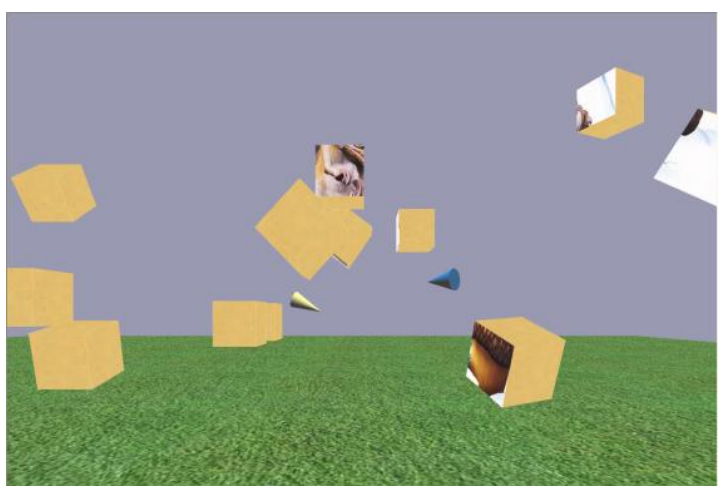

Figure 2. The puzzle solving task used in the first experiment.

\subsection{Results}

In this article, we focus on improving group interaction in a collaborative virtual environment by providing adaptation of assigning users' roles based on a collaborative user model. The collaborative user model is built based on the users' individual performance and gives information about the best combination of roles and devices for a particular group. To investigate the efficacy of the model, suggested in Section 4, we compared the estimated task completion time based on this model with the actual task completion time of that particular combination. Furthermore, we found it interesting to compare the model and actual time with the task completion time measured during the free collaborative session, in order to see whether or not implementing adaptation for role-dependent collaboration would significantly decrease task completion time, thus enhance group interaction.

We employed the proposed 'minimum time' approach to determine the best role combination as the collaborative user model. In this approach, we estimated the task completion time by first estimating the time to perform each role per cube for every user (e.g. to translate one cube with SpaceMouse). Then for all combinations of roles and devices, we calculated the total time to rotate and translate one cube. Afterwards we estimated the total time to complete the whole task collaboratively. As a result, we determined the minimum estimated total time which then defined the best role combination as well.

For the first hypothesis, a paired-samples t-test showed no significant difference between the model time and the actual time [ $\mathrm{t}(9)=0.212, \mathrm{p}=0.837$ ]. This indicates that task completion times do not differ significantly across the model and actual times. Based on this finding, we can conclude that 
the constructed model can be considered as a good approximation to the actual condition. As no difference across model time and actual time was found, we can conclude that we have successfully selected the right combination of roles and devices, indicating the model's efficiency.

For the second and third hypotheses, pairedsamples t-tests showed statistically significant differences between the model time and the free time $[\mathrm{t}(9)=3.508, \mathrm{p}=0.007]$, and also between the actual time and the free time $[\mathrm{t}(9)=2.302, \mathrm{p}=$ 0.047]. These findings indicate task completion times differ significantly across the role-dependent collaboration (model and actual) and the freecollaboration times. The average task completion times for cases where roles were introduced (model $[\mathrm{M}=217.8 \mathrm{~s}]$, actual $[\mathrm{M}=213.9 \mathrm{~s}]$ ) were lower than for cases where roles were not present (free $[\mathrm{M}=$ $283.5 \mathrm{~s}]$ ). These results indicate that assigning roles to users improves the group performance.

\section{Experiment II: Adaptation in Multiplayer Video Games}

In the previous section we have shown that providing adaptation based on the user performance (in form of assigning users with a certain role and device) leads to improved group interaction in a general collaborative virtual environment. An experiment was conducted in a fully controlled environment where two users had to complete a puzzle solving task that consisted of two basic manipulations: translation and rotation.

However, in real-life collaborative applications, the task division does not happen at such atomic level. Usually each collaborator has a number of tasks that correspond to him/her and that he/she is responsible for. Therefore, as a next step, we aim to investigate the potential of role-based adaptation according to the user context in a more realistic scenario. As an example of such scenario, we have chosen multiplayer video games. In some existing collaborative games, task division and role playing become important to improve the collaboration and win the game at the end. For example in Battlefield games ${ }^{5}$, the players need to divide their tasks when one player has to control the vehicle while the other is responsible for shooting the enemies. In the Little Big Planet game ${ }^{6}$, sometimes the players need to closely collaborate to overcome certain obstacles which can only be moved away when players do this at the same time.

Little research has investigated ways of improving collaboration in games through the act of dividing tasks between players. Therefore, we intend to investigate the assignment of specific roles to

\footnotetext{
${ }^{5}$ http://www.battlefield.com

${ }^{6} \mathrm{http}: / /$ www.littlebigplanet.com
}

players based on their individual characteristics (i.c. performance) as a form of adaptation in collaborative games. Furthermore, due to the differences that exist between applications designed for research experiments (like the one used for the first experiment) and real video games, we aim to outline some of the challenges that occur when applying the modeling approach presented earlier in Section 4 in the context of games.

In order to achieve the aforementioned goals, a user experiment was conducted. For the experiment, a recent commercial game Trine was chosen, which involves several characters, each playing specific role, and requires collaboration between them in order to complete the game. Similar to the case of general virtual environments, the same type of adaptation based on assigning users' roles and devices has been considered.

\subsection{Hypotheses}

During the second experiment, the primary focus was on the applicability of the proposed 'minimal time' approach for adaptation in video games. Therefore, here, we only checked hypothesis (1), which validated the proposed approach: the estimated task completion time by each pair of the participants based on the model will not differ from the actual task completion time. Hypotheses (2) and (3) are not considered here, as the game chosen for the experiment does not support collaboration without roles.

\subsection{Participants}

Eight pairs of randomly coupled unpaid participants (two females and fourteen males) participated in the experiment. The average age of the participants was 28 years old, varying from 22 till 36 years. All participants were recruited among the university staff and had a computer science background. Part of the subjects participated in the first experiment presented in Section 5.

\subsection{Apparatus}

For the experiment, two different setups were used: one for the individual session and the other for the collaborative session. During the individual session two separate laptops with 15 inches widescreens were involved, each used by a different player. For the collaborative sessions one laptop was connected to a 40 inches screen, which was shared by two players. A keyboard with mouse and the Xbox controller were used as the input devices. The setup of a collaborative session is shown on Figure 3.

\subsection{Design and procedure}


During the second experiment, each pair of the participants was asked to complete two sessions: an

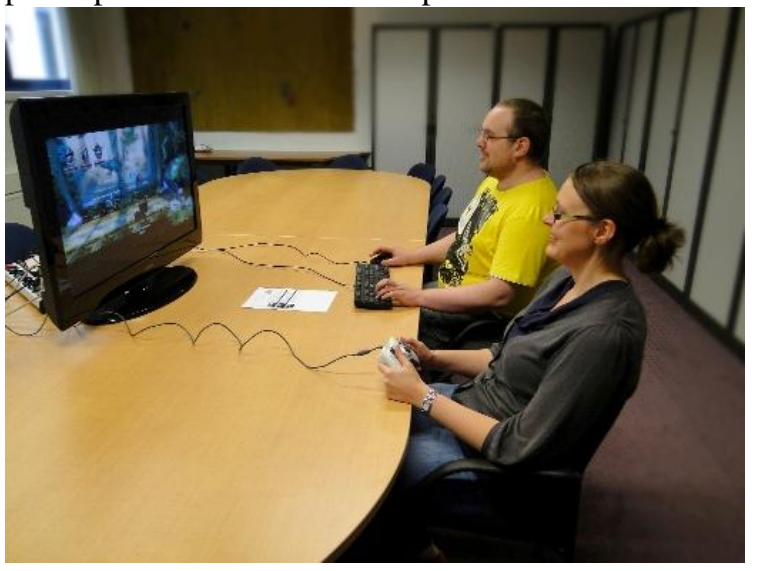

Figure 3. The setup in the second experiment.

individual session and a role-dependent collaborative session. Similarly to the experiment in a general virtual environment, data collected during the individual session was used for constructing a model, which was validated by comparison with an actual role-based performance collected during the collaborative session.

In the game Trine, a commercially available game used for this experiment, each player is represented as one of three characters: the thief, the knight or the wizard. Each character has a certain set of actions available only to him. Therefore, to complete the game collaboration of all characters is needed.

To interact with the character and the environment players used one of the following devices: keyboard with mouse or Xbox controller. Similarly to the first experiment, a within-subject design was applied for the collaborative sessions, where the independent variable was the combination of role and device. The task completion time was measured as the dependent variable. Table 2 presents the complete overview of the experiment sessions.

Table 2. Sessions in the second experiment (Trine).

\begin{tabular}{|c|c|c|}
\hline & Participant 1 & Participant 2 \\
\hline \multirow{4}{*}{ 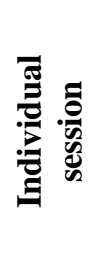 } & Thief Keyboard & Thief Keyboard \\
\hline & Knight Keyboard & Knight Keyboard \\
\hline & Thief Xbox controller & Thief Xbox controller \\
\hline & Knight Xbox controller & Knight Xbox controller \\
\hline \multirow{4}{*}{ 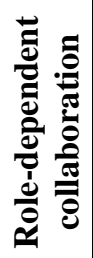 } & Thief Keyboard & Knight Xbox controller \\
\hline & Knight Keyboard & Thief Xbox controller \\
\hline & Thief Xbox controller & Knight Keyboard \\
\hline & Knight Xbox controller & Thief Keyboard \\
\hline
\end{tabular}

To avoid an excessive number of test conditions and to have a similar pair-based experiment as in Section 5, only two roles were involved during the experiment (thief and knight). During the individual session, every participant had to complete two short levels, each introducing abilities of these two characters. Participants had to perform these levels twice: once using keyboard with mouse, and then using an Xbox controller.

During the collaborative session participants were asked to complete the actual game level, where all game characters were involved. The same level was played four times, involving all possible combinations of two roles and two devices (see Table 2). We did not involve a third person to play as the wizard. However, players were allowed to switch to the wizard character only when it was not possible to progress within the level otherwise. Afterwards, players were obliged to switch back to the character they were playing. An example of a screenshot of the gaming environment with two characters is shown in Figure 4. It took approximately 1 hour for each pair to complete the entire experiment.

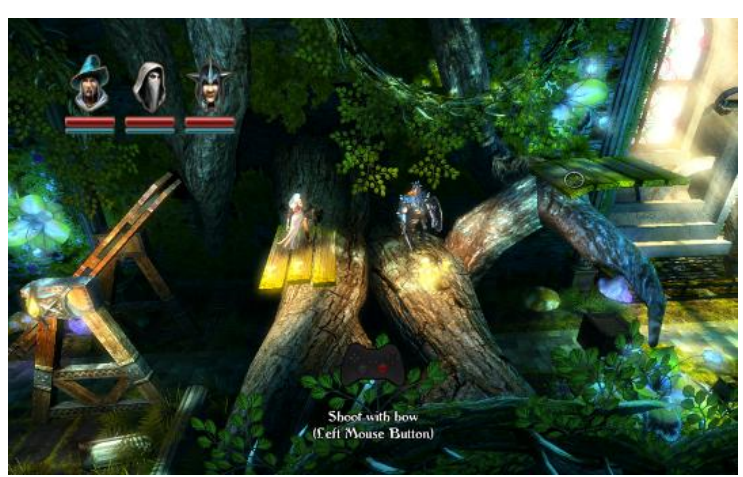

Figure 4. The gaming environment used in the second experiment.

\subsection{Results}

As stated earlier, with the help of a user experiment we wanted to investigate to what extent the approach suggested in Section 4 for a controlled virtual environment is applicable in a real-life scenario. In particular, we chose the domain of video games as one of the most popular types of collaborative virtual environments. With an attempt to apply the proposed approach in an existing video game, we aim to identify some challenges that occur when introducing role-based adaptation in video games. Therefore, we present our results in two steps. First, we describe the challenges arising when applying the modeling approach presented in Section 4. Secondly, we report on the validation of the model for a video game.

One of the immediate challenges that we have encountered when applying the 'minimal time' 
approach, was the modeling itself. In accordance with the presented approach, we calculated the model time based on the user performance. In case of the puzzle solving experiment, we used the same task for both individual and collaborative sessions (every puzzle task involved the same amount of puzzle blocks during individual and collaborative sessions). In case of the video games, it might not be always possible to use the same game level in order to determine the most suitable role for each player. One possible reason for this is the learning of the game levels. This, in turn, may lead to a better performance due to the knowledge on how to solve puzzles or overcome obstacles within the level, rather than due to the provided adaptation. Moreover, some games involve several characters that play different, often complementary, roles (similar to Trine used for the experiment). In such games, the levels might be designed in such a way that it is often impossible to complete it on your own using only one specific character or role.

These considerations trigger a necessity to search for an alternative that can be used for acquisition of individual data. When analyzing modern video games, one may notice that many of them force players to complete (usually, short and easy) trial level(s) before the actual gameplay. During such trial levels, players learn main elements that occur further during the game. As the game Trine requires players to complete the trial levels and to gain a certain experience level, they were used to obtain the data regarding the individual performance during our experiment.

However, using trial levels raises another issue for the modeling of the user performance. As mentioned earlier, trial levels of the existing games are usually easier than the real game levels. In particular, in the game being used a trial level contains only one obstacle and the distance that players pass is almost half of the one used in the actual level. In this case, applying modeling based on one parameter (either amount of obstacles, or distance) would lead to incorrect time estimation. Therefore, in our experiment, both distance, passed by each player, and the amount of obstacles that appeared along this distance have been taken into account to model the player performance.

After adjusting the modeling approach according to the aforementioned considerations, we followed the same steps as in the first experiment with the puzzle solving task. The model consists of the variables that represent the combinations of roles and devices that resulted in the shortest task completion. To validate the constructed model, we compared modeled time with the one in a collaborative session (values are selected for the same combinations of devices and roles).

To validate the modeling approach in case of the video game we compared modeled and actual time (hypothesis 1). A paired-samples t-test showed no significant difference among them $[\mathrm{t}(7)=0.303 \mathrm{p}=$ $0.77]$, indicating that our model $[\mathrm{M}=481.40]$ can be considered as a good approximation to the actual [M $=498.38$ ] condition. The obtained results indicate the effectiveness of the proposed modeling approach (based on the amount of obstacles and distances passed within the game) to determine the right combination of roles and devices for the commercial game being used in the experiment.

\section{Conclusion}

We presented an investigation of context-based adaptation as an approach to enhance group interaction in collaborative virtual environments and in multiplayer video games. First, we have described an experiment to examine how adaptation according to the context of users (i.e. users' role and device used) leads to improved group collaboration (i.e. faster task completion time) in virtual environments. We found that assigning roles to users, as described by our model, improves the group performance.

Secondly, we have applied the same approach to provide adaptation in a multiplayer video game to investigate its potential in a real-life scenario. Based on the example of a recent commercial game Trine, we have revealed some of the challenges that occurred when applying adaptation of assigning roles to users in this game. One of these challenges was the necessity to adjust the modeling approach to estimate the completion time (model time). We have shown that for the modeling it is important to take into account task related information, in our case the amount of manipulated blocks in the collaborative virtual environment and the amount of obstacles and distance in the game. Considering this task specific parameterization, the 'minimal time' approach has provided enhanced group collaboration.

In this article we have considered only few types of collaboration in virtual environments and games. Further investigation is absolutely necessary to verify whether or not the proposed 'minimum time' modeling approach is working for other collaborative tasks. Additionally, the possibility to integrate the proposed approach for a run-time adaptation needs a thorough investigation.

\section{References}

[1] C. Mourlas and P. Germanakos. Intelligent User Interfaces: Adaptation and Personalization Systems and Technologies. Information Science Reference, 2009.

[2] A. K. Dey. Understanding and using context. Personal and Ubiquitous Computing, 5(1):4-7, 2001.

[3] W. Höpken, M. Scheuringer, D. Linke, and M. Fuchs. Context-based adaptation of ubiquitous web applications in 
tourism. In Information and Communication Technologies in Tourism 2008, pages 533-544. Springer Vienna, 2008.

[4] A. Celentano, M. Nodari, and F. Pittarello. Adaptive interaction in web3d virtual worlds. In Proceedings of the 9th International Conference on 3D Web Technology, pages 41-50, 2004.

[5] J. R. Octavia, L. Vanacken, C. Raymaekers, K. Coninx, and E. Flerackers. Facilitating adaptation in virtual environments using a context-aware model-based design process. In Proceedings of the 8th International Workshop on Task Models and Diagrams for User Interface Design, volume 5963 of LNCS, pages 58-71. Springer, 2010.

[6] Hariandja, J. R. O. Adaptivity in virtual environments: enhancing user interaction and accommodating user diversity. PhD thesis, Hasselt University, 2011.

[7] S. J. Kim, K. J. Gil, H. Kim, S. B. Lim, and J.-i. Kim. Adaptive interactions in shared virtual environments for heterogeneous devices. Computer Animation and Virtual Worlds, 21(5):531-543, 2010.

[8] G. N. Yannakakis. Game AI revisited. In Proceedings of the 9th conference on Computing Frontiers, pages 285292. ACM, 2012.

[9] R. Hunicke. The case for dynamic difficulty adjustment in games. In Proceedings of the 2005 ACM SIGCHI International Conference on Advances in computer entertainment technology, pages 429-433. ACM, 2005.

[10] G. N. Yannakakis, and J. Hallam. Towards optimizing entertainment in computer games. Applied Artificial Intelligence, 21(10):933-971, 2007.

[11] F. Buttussi, L. Chittaro, R. Ranon, and A. Verona. Adaptation of graphics and gameplay in fitness games by exploiting motion and physiological sensors. In A. Butz, B. Fisher, A. Krüger, P. Olivier, S. Owada, editors, Smart Graphics, volume 4569 of Lecture Notes in Computer Science, pages 85-96. Springer Berlin / Heidelberg, 2007.

[12] R. Schroeder, A. Steed, A.-S. Axelsson, I. Heldal, A. Abelin, J. Wideström, A. Nilsson, and M. Slater. Collaborating in networked immersive spaces: as good as being there together? Computers \& Graphics, 25(5):781 788, 2001.

[13] A. Steed, M. Slater, A. Sadagic, A. Bullock, and J. Tromp. Leadership and collaboration in shared virtual environments. In Proc. IEEE Virtual Reality, pages 112115. IEEE Computer Society, 1999.

[14] A. Beznosyk, C. Raymaekers, K. Coninx, P. Quax, and W. Lamotte. Investigating the influence of communication and input devices on collaboration in virtual environments. In Proceedings of the 5th International Conference on Computer Graphics Theory and Applications, pages 341-346, 2010.

[15] M. Kirsch-Pinheiro, M. Villanova-Oliver, J. Gensel, and $\mathrm{H}$. Martin. Context-aware filtering for collaborative web systems: adapting the awareness information to the user's context. In Proc. SAC, pages 1668-1673. ACM, 2005.

[16] S. Bergsträsser, T. Hildebrandt, L. Lehmann, C. Rensing, and R. Steinmetz. Virtual context based services for support of interaction in virtual worlds. In Proceedings of the 6th ACM SIGCOMM workshop on Network and system support for games, pages 111-116. ACM, 2007.

[17] A. Singh and A. Acharya. Using session initiation protocol to build context-aware VoIP support for multiplayer networked games. In Proceedings of 3rd ACM SIGCOMM workshop on Network and system support for games, pages 98-105. ACM, 2004.

[18] S. Gerbaud, V. Gouranton, and B. Arnaldi. Adaptation in collaborative virtual environments for training. In Learning by Playing. Gamebased Education System Design and Development, volume 5670 of Lecture Notes in Computer Science, pages 316-327. Springer Berlin / Heidelberg, 2009.

[19] Ibáñez, J. and Delgado-Mata, C. Adaptive two-player videogames. Expert Systems with Applications, 38(8):9157-9163, 2011.

[20] J. R. Octavia, A. Beznosyk, K. Coninx, P. Quax, and K. Luyten. User modeling approaches towards adaptation of users' roles to improve group interaction in collaborative $3 \mathrm{~d}$ games. In Proceedings of the 14th international conference on Human-computer interaction: users and applications - Volume Part IV, pages 668-677, Springer-Verlag, 2011. 